\title{
Broadband wide-angle polarization converter for LCD backlight
}

\author{
Chang-Ching Tsai* and Shin-Tson Wu \\ College of Optics and Photonics, University of Central Florida, Orlando, Florida 32816, USA \\ ${ }^{*}$ Corresponding author: tsaicc@ @creol.ucf.edu
}

Received 18 April 2008; accepted 24 April 2008;

posted 29 April 2008 (Doc. ID 95136); published 15 May 2008

\begin{abstract}
A novel polarization converter using reflective metallic gratings and a polarization beam splitter is introduced for LCD backlight illumination. These two optical elements form a polarization rotation resonator. Broadband and high optical efficiency of polarization conversion in the visible region is achieved through the resonance of the refracted light and the surface plasmon wave in metallic surface-relief gratings. For wide-angle illumination, the conversion efficiency with arbitrary incident angle is studied. This device can convert unpolarized light to linear polarization with over 85\% efficiency. @ 2008 Optical Society of America

OCIS codes: $\quad 230.5440,240.6680,050.6624$.
\end{abstract}

\section{Introduction}

Two crossed linear polarizers are commonly used in direct-view liquid crystal displays (LCDs) in order to achieve a high contrast ratio [1]. However, under such a device configuration the maximum transmittance is reduced to $\sim 45 \%$ because of the absorption of the sheet polarizers. Several approaches for converting randomly polarized light into linear polarization have been developed [2]. Among them, the dual brightness enhancement film (DBEF) developed by $3 \mathrm{M}$ has been widely utilized [3]. In DBEF, a polarizing beam splitter (PBS) and a diffusive reflector are employed to recycle the reflected polarization component. In recycling, first the PBS selects one polarization, say TM, to pass through and reflects the TE wave to the diffusive reflector (DR). Second, through hitting the rough surface of $\mathrm{DR}$, the reflected light is randomly unpolarized and sent back to PBS again. As a result, $\sim 40 \%$ of the unused light is recovered by many such recycles. However, the recycling efficiency is limited in this configuration due to deficient polarization conversion by DR. Therefore, there is an urgent need to develop a more proficient polarization

0003-6935/08/152882-06 $\$ 15.00 / 0$

(C) 2008 Optical Society of America converter in order to reduce the power consumption of LCDs.

The function of depolarizing linear polarized light by a diffusive reflector is through multiple irregular reflections changing various propagating directions, hence the polarizations, on the coarse DR surface. Averagely speaking, no more than $50 \%$ of TE light will be rotated into TM with the DR. One highly efficient polarization conversion scheme for a reflected beam is through the resonance of incident light and the induced surface plasmon wave on metallic gratings. Such surface-plasmon-aided polarization conversion was first experimentally observed by Bryan-Brown and Sambles [4] in silver-coated sinusoidal surface-relief gratings, where near $9 \%$ of polarization conversion was recorded. The excitation of a surface plasmon wave is actually due to the conical diffraction at a $45^{\circ}$ azimuthal angle, which will cause the reflected light to be rotated into elliptic polarization to a certain degree. In some specific grating configurations, above $70 \%$ polarization rotation of a TM (or TE) wave into TE (or TM) for wavelengths within $550-900 \mathrm{~nm}$ is possible [5]. The conversion efficiency depends on the details of how the surface plasmon is coupled into photons (optical modes) and how these photons interfere with the diffracted light. This coupling depends critically on the groove shape and 


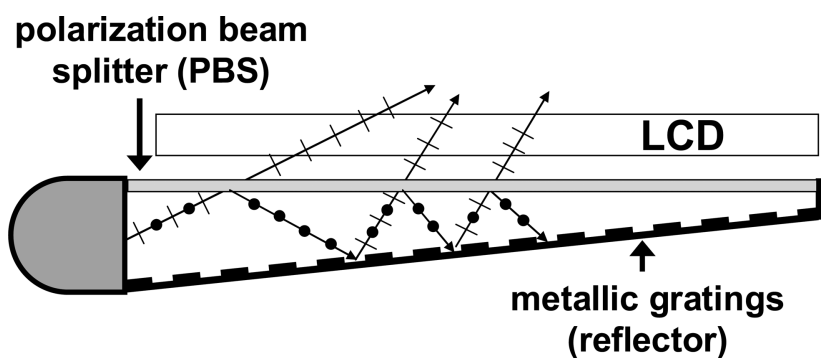

Fig. 1. Light recycling system for LCD backlight illumination, where the unpolarized light passes one linear polarization through the polarization beam splitter and the other polarization is directed to the polarization converting reflector.

depth of gratings. More detailed analysis on such surface-plasmon-aided polarization rotation can be found in [6-10].

In the early studies of metallic gratings, only monochromatic light was discussed. This indeed limits its application to broadband light sources. Later, several types of metallic gratings were reported that enable broadband polarization conversion in the visible spectral regions [5,11]. With this nice polarization conversion efficiency and high reflectivity of some metallic gratings, it is possible to replace the diffusive reflector in the light recycling system. However, except the remarkable progress in achromatic performance, the above mentioned reports only concerned single incident-angle light. To apply the metallic gratings to general light sources, such as
Lambertian for an LCD's backlight illumination, the study of large polarization conversion with arbitrary incident angle is necessary.

Here, we investigate the wide-angle performance of broadband polarization conversion of specific metallic gratings and propose a new light recycling scheme. The multibouncing between PBS and metallic gratings to enhance the total transmittance of polarization-converted light is numerically studied here. A conversion efficiency higher than $85 \%$ is achieved in our design.

\section{Device Configuration and Operation Mechanisms}

As described in Ref. [10], to have a broadband polarization conversion, it needs high modulated surfacerelief gratings, i.e., the groove depth $d$ is at least one quarter of the wavelength, $d \geq \lambda / 4$. Recently, three types of subwavelength metallic gratings, Gaussianridge, binary, and trapezoidal profiles, have been reported [12] to have high polarization conversion centered in the entire visible spectrum. For simplicity and feasibility of manufacture, only the trapezoidal silver grating is considered here in wide-angle analysis. The light recycling system is depicted in Fig. 1 . The multibouncing between the PBS and trapezoidal grating inside the polarization-rotating-resonator is illustrated in Fig. 2. The trapezoidal grating profile is also shown in Fig. $\overline{2}$ with the trapezoid top $60 \mathrm{~nm}$, bottom $70 \mathrm{~nm}, d=12 \overline{4} \mathrm{~nm}$, and pitch $\Lambda=197 \mathrm{~nm}$.

In Fig. 1, we see that the unpolarized light is coming out from the edge illuminating source and guided

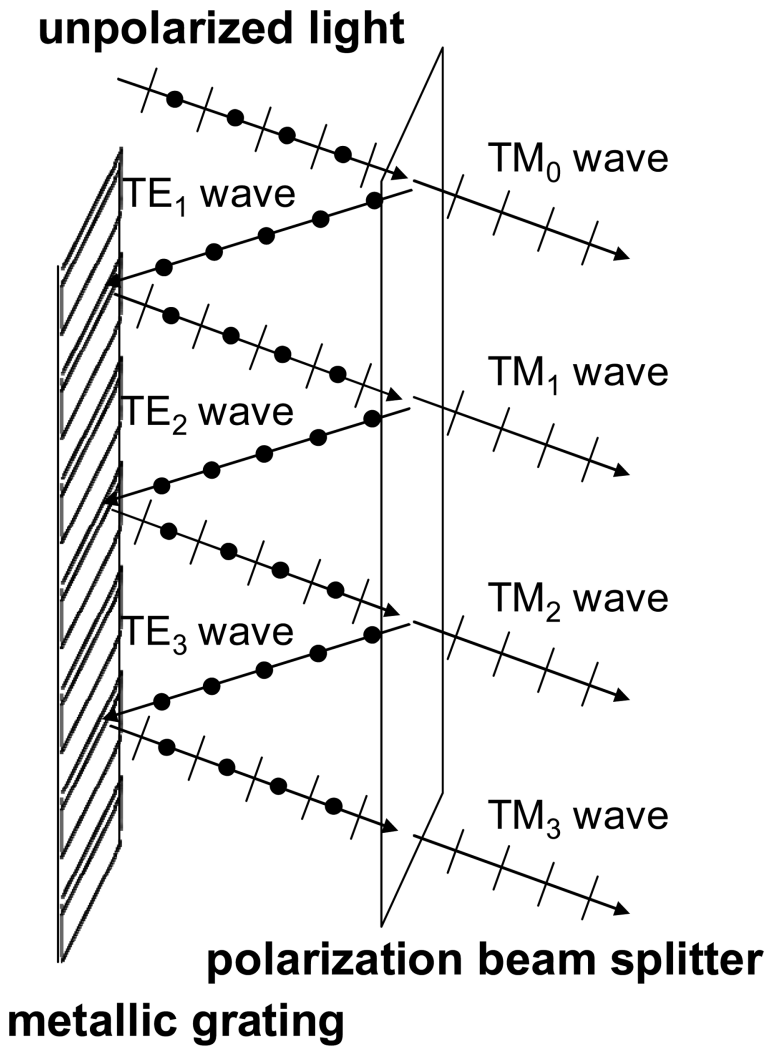

$$
\begin{aligned}
& \mathbf{T M} \mathbf{t o t a l} \\
& =\mathbf{T M}_{0}+\mathbf{T} \mathbf{M}_{1}+\cdots+\mathbf{T} \mathbf{M}_{\infty} \\
& \mathbf{T} \mathbf{M}_{0}=1-\mathbf{T E} \mathbf{E}_{1} \\
& \mathbf{T} \mathbf{M}_{i}=\boldsymbol{c} \times \mathbf{T E} \mathbf{E}_{i} \\
& \mathbf{T E _ { i + 1 }}=(\boldsymbol{r}-\boldsymbol{c}) \times \mathbf{T E} \mathbf{i}_{i} \\
& \boldsymbol{r}: \text { reflectance } \\
& \boldsymbol{c}: \text { conversion efficiency } \\
& \boldsymbol{i}: \text { cycle number }
\end{aligned}
$$

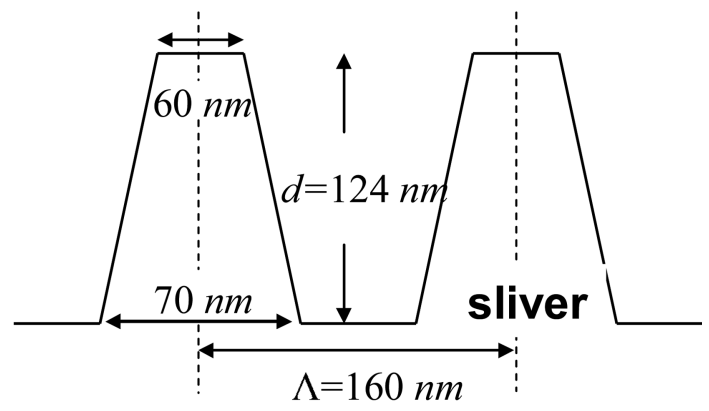

trapezoidal grating profile

Fig. 2. Recycling process of linear polarized light through multibouncing between the polarization beam splitter and metallic grating reflector; also shown is the profile of sliver trapezoidal grating. 
into the recycling region sandwiched between the PBS and the reflector. As mentioned in the introduction section, we will now replace the reflective diffuser with metallic gratings as the reflector. Figure 2 shows the recycling process as follows. The TM $(\bar{p}$ wave) component of the initial incident unpolarized light passes through the PBS, while the TE ( $s$ wave) part is reflected toward the metallic grating. The metallic grating rotates the incoming $\mathrm{TE}$ linearly polarized light into an elliptical polarized wave. After the rotation the new TM component will go through the PBS and the remaining TE component will be redirected onto metallic grating again. This is called one cycle of polarization conversion. During one cycle, the PBS selects one polarization and the metallic grating rotates the other, respectively. Both should work very well in broadband spectrum and wide angular range. The subwavelength trapezoidal grating described in Fig. 2 and the PBS in Ref. [3] both feature the capability. The system configuration is similar to a Fabry-Perot resonator where the total transmittance is obtained by adding up all the rays bouncing out of the resonator. Similarly, after infinite cycles of polarization conversion, the total conversion efficiency is greatly enhanced.

In a Fabry-Perot resonator, the wave propagation is in the geometric optics regime, and the ray bouncing back and forth inside the resonator follows Snell's law. It is straightforward to calculate the total transmittance once the reflectance and transmittance on each boundary are known. In our design, although diffraction optics should be considered here, the same principle also applies. This is because our subwavelength grating allows only the zeroth-order diffracted wave to propagate. The others are the evanescent waves. Hence, the wave bouncing inside the resonator also follows Snell's law. This nice property enables us to calculate the result of each incidentangle ray individually after infinite bounces, and the summation of each result gives us the total polarization conversion by the superposition principle. The total conversion intensity can be expressed as follows:

$$
\begin{aligned}
\mathrm{TM}_{0} & =1-\mathrm{TE}_{1}, \quad \mathrm{TM}_{i}=\boldsymbol{C}_{s p} \times \mathrm{TE}_{i}, \\
\mathrm{TE}_{i+1} & =\left(\boldsymbol{R}-\boldsymbol{C}_{s p}\right) \times \mathrm{TE}_{i} \\
\mathrm{TM}_{\text {total }} & =\mathrm{TM}_{0}+\mathrm{TM}_{1}+\ldots+\mathrm{TM}_{\infty} \\
& =1+(\boldsymbol{R}-1) /\left(1+\boldsymbol{C}_{s p}-\boldsymbol{R}\right) \times \mathrm{TE}_{1},
\end{aligned}
$$

where $\boldsymbol{C}_{s p}$ is the conversion efficiency for an $s$ wave converted into a $p$ wave, $\boldsymbol{R}$ is the reflectivity of the grating, and $i$ is the cycling number. Here the definition of $\boldsymbol{C}_{s p}$ is the power carried by the reflected $p$ wave divided by that of the incident $s$ wave. Typically for a normalized randomly polarized light, we set the intensity $\mathrm{TE}_{1}=\mathrm{TM}_{0}=0.5$.

For effective polarization conversion, it needs the incident plane neither parallel nor normal to the grating vector, i.e., the azimuthal angle $\phi$ (the angle between incident plane and gratings vector) should

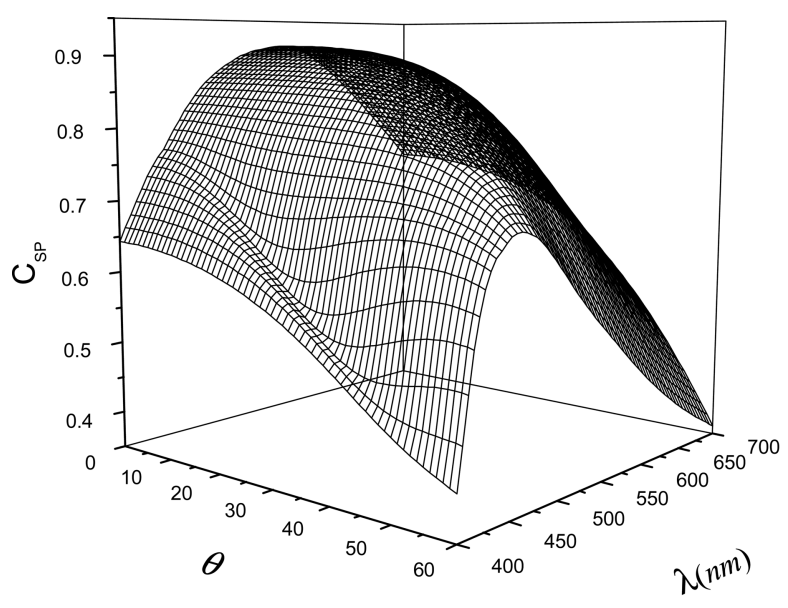

(a)

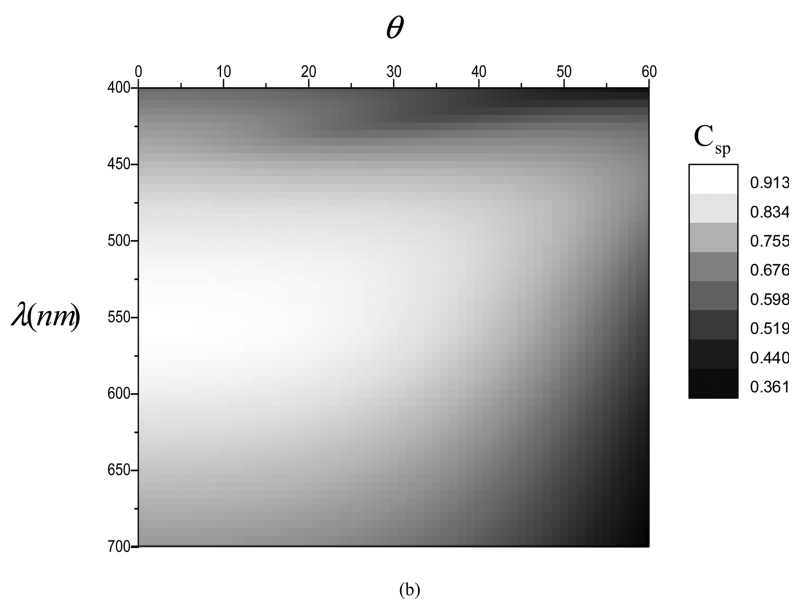

Fig. 3. (a) Polarization conversion efficiency $\boldsymbol{C}_{s p}(\lambda, \theta)$ without light recycling of the trapezoid grating for $\phi=45^{\circ}$ with maximum $\boldsymbol{C}_{s p}=91.3 \%$, minimum $\boldsymbol{C}_{s p}=36.1 \%$, and average $\overline{\boldsymbol{C}}_{s p}=75.7 \%$. (b) Top view of (a).

be $0<\phi<90^{\circ}$. In shallow gratings, where the groove depth is much less than the pitch $d \ll \Lambda$, conversion efficiency $\boldsymbol{C}_{s p}$ follows the square rule of a sinusoidal function, $C_{p s}(\theta, \phi) \propto \sin ^{2}(2 \phi)$, which was both empirically and analytically proven $[1,13]$. In the present broadband cases, we need high aspect ratio gratings with $d>\Lambda$ or at least $d \approx \Lambda$ to have high $\boldsymbol{C}_{s p}$. For such deep gratings, the physics of the surface plasmon wave and diffracted light is very complicated, since the excited optical modes of surface plasmons can have self-interferences inside the groove or form complex bandgap structures [14]. Therefore, the numerical analysis of conical diffraction with metallic gratings is necessary to determine the optimized $\boldsymbol{C}_{s p}$ condition with variants of incident angle $\theta$, azimuthal angle $\phi$, and wavelength $\lambda$.

Here we will briefly discuss the numerical schemes used in calculating polarization conversion. A common approach to model the light with metallic gratings is based on the coordinate transformation technique developed by Chandezon et al. [15] and Elston et al. [6]. Other researchers alternately used 
Yasuura's mode-matching method $[16,17]$ to calculate the $p$-s mode conversion by metallic grating. Another popular way in calculating diffraction gratings is the conical rigorous coupled-wave analysis (RCWA) proposed by Moharam and Gaylord [18,19]. Several results using RCWA to calculate the polarization conversion have been reported $[20,21]$. In the present work, we will employ RCWA to investigate the conversion performance of deep metallic gratings. Here we will not address the details of how to formulate RCWA in doing the calculation. Actually, one feasible way of obtaining the polarization conversion by RCWA with separate $s$ and $p$ modes represented in the reference frame of incident plane was recently reported in Ref. [12].

\section{Numerical Results of Broadband Wide-Angle Polarization Converter}

We will first demonstrate the $\boldsymbol{C}_{s p}(\lambda, \theta)$ performance of the silver trapezoidal gratings in Fig. 2. The simulation condition is at fixed azimuth angle $\phi=45^{\circ}$ with $\theta$ ranging from 0 to $60^{\circ}$ and $\lambda$ ranging from 400 to $700 \mathrm{~nm}$. During simulation, both the dispersion and absorption of silver in the visible region from ex-
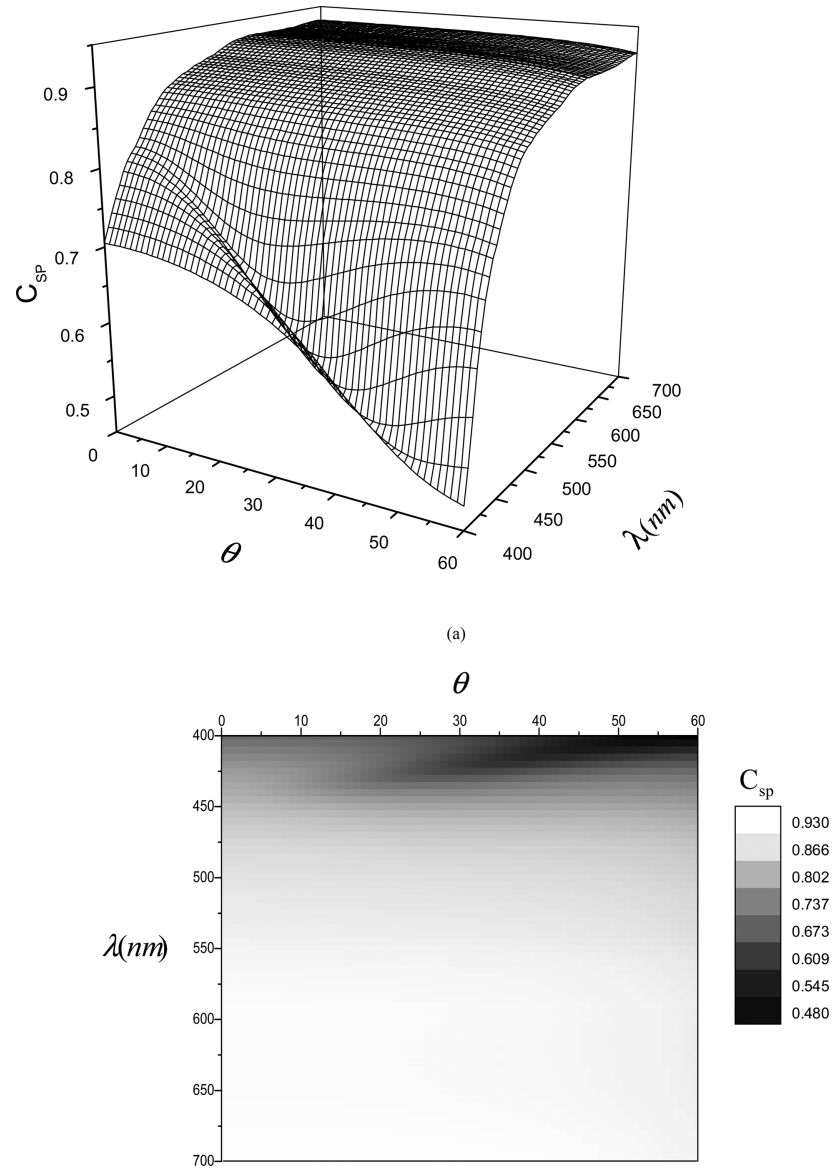

(b)

Fig. 4. Enhanced polarization conversion efficiency $\boldsymbol{C}_{s p}(\lambda, \theta)$ with light recycling of the same grating and incident condition as in Fig. 3. (a) Maximum $C_{s p}=93.0 \%$, minimum $C_{s p}=48.0 \%$, and average $\overline{\boldsymbol{C}}_{s p}=87.2 \%$. (b) Top view (a). periment data [22] are used in the calculation. The result is shown in Fig. 3(a) and 3(b), where we can see in the major region centered around $\lambda=$ $550 \mathrm{~nm}$ that the $\boldsymbol{C}_{s p}$ is more than $75 \%$. This is critical for LCD applications, since the human eye is most sensitive to green light. This proves that without recycling, our trapezoid grating still gives reasonable conversion efficiency at wide-angle incidence. Next, we will see the effective $\boldsymbol{C}_{s p}$ after infinite ray bounces in the recycling case by calculating the $\mathrm{TM}_{\text {total }}$ term in Eq. (1). The loss of rays passing the PBS are ignored here, since the absorption of the PBS is often negligibly small [3]. The result is shown in Fig. 4(a) and $4(\mathrm{~b})$. Clearly, the final $C_{s p}$ can reach above $90 \%$ after infinite cycles in most of the $(\lambda, \theta)$ region. This manifests that the conversion efficiency is greatly improved by use of this resonator structure. For more quantitative description, we define the figure of merit,
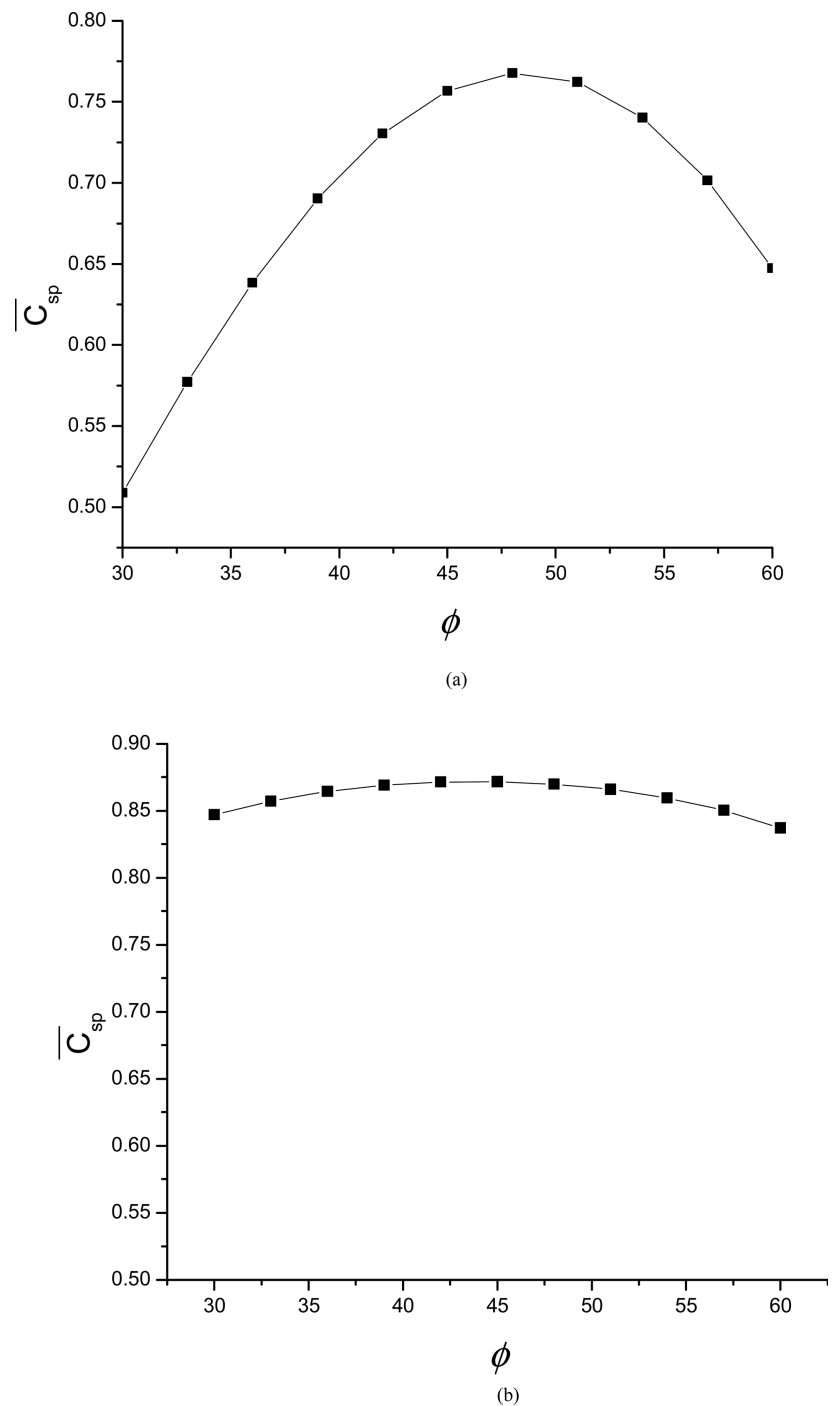

Fig. 5. (a) $\overline{\boldsymbol{C}}_{s p}(\phi)$ curve without light recycling of trapezoid grating for $\phi=30^{\circ}$ to $60^{\circ}$, the maximum $\overline{\boldsymbol{C}}_{s p}=76.7 \%$ occurs at $\phi=48^{\circ}$. (b) Enhanced $\overline{\boldsymbol{C}}_{s p}(\phi)$ curve with light recycling; the maximum $\overline{\boldsymbol{C}}_{s p}=87.2 \%$ occurs at $\phi=45^{\circ}$. 

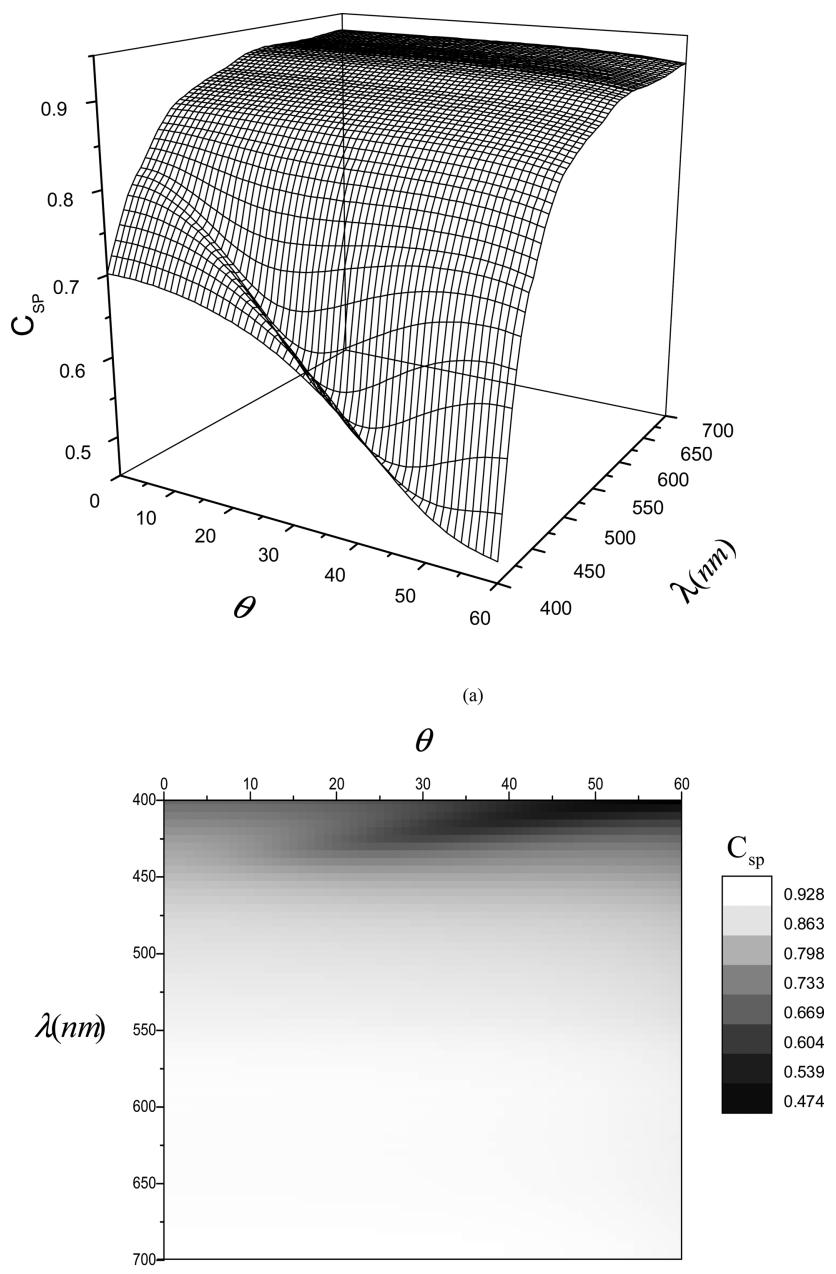

(b)

Fig. 6. (a) Enhanced polarization conversion efficiency $\boldsymbol{C}_{s p}(\lambda, \theta)$ with light recycling of the trapezoid grating for $\phi=48^{\circ}$ with maximum $\boldsymbol{C}_{s p}=92.8 \%$, minimum $\boldsymbol{C}_{s p}=47.4 \%$, and average $\overline{\boldsymbol{C}}_{s p}=87.0 \%$. (b) Top view of (a).

$$
\overline{\boldsymbol{C}}_{s p}=\iint \boldsymbol{C}_{s p}(\lambda, \theta) \mathrm{d} \lambda \mathrm{d} \theta / \Delta \lambda \Delta \theta,
$$

as the average $\boldsymbol{C}_{s p}$ over the incident angle and wavelength ranges. We have $\overline{\boldsymbol{C}}_{s p}=75.5 \%$ without recycling in Figs. 3(a) and $3(\mathrm{~b})$ and $\overline{\boldsymbol{C}}_{s p}=87.2 \%$ for infinite cycles in Figs. 4(a) and 4(b).

Now we will explore the dependence of $\overline{\boldsymbol{C}}_{s p}$ on the azimuthal angle $\phi$. Here we plot $\overline{\boldsymbol{C}}_{s p}(\phi)$ for both with and without the recycling in Figs. $\underline{5}$. In Fig. $\underline{5(\mathrm{a})}, \overline{\boldsymbol{C}}_{s p}(\phi)$ is without recycling, which shows that the maximum $\overline{\boldsymbol{C}}_{s p}=76.7 \%$ occurs at $\phi=48^{\circ}$. It is then natural to assume that the optimized $\overline{\boldsymbol{C}}_{s p}(\phi)$ for infinite conversion cycles will also happen at $\phi=48^{\circ}$. However, after the calculation we found that with recycling in Fig. 5(b) the optimized $\overline{\boldsymbol{C}}_{s p}=87.2 \%$ occurs at $\phi=45^{\circ}$. In fact, Fig. $5(\mathrm{~b})$ shows the $\overline{\boldsymbol{C}}_{s p}(\phi)$ curve is pretty flat and not so sensitive to the azimuth angle. In the range of $\phi=$ $30^{\circ}$ to $50^{\circ}$, the conversion efficiencies are pretty similar. We then further plot the recycling $C_{s p}(\lambda, \theta)$ for $\phi=$ $48^{\circ}$ in Figs. 6(a) and 6(b). Comparing Figs. 4 and 6 as these two figures show, both $C_{s p}$ for $\phi=45^{\circ}$ and $48^{\circ}$ distribute uniformly in the main region of $(\lambda, \theta)$, and the deviations of each maximum and minimum $\boldsymbol{C}_{s p}$ are pretty much the same. This provides an additional advantage to our design that in real applications the precision of azimuthal alignment is not highly required.

\section{Conclusion}

We have demonstrated a broadband wide-angle polarization converter for LCD applications. Simulation results show our device featuring both high spectral and angular performances. The silver trapezoid grating given here is only an example for working in the visible light region. Indeed, other types of surface-relief gratings or materials may give similar or better performance. Compared with the current design of reflective diffuser, our recycling scheme can save much more illuminating power. Especially, it would greatly benefit those portable devices, where power consumption is always a critical issue.

The authors are indebted to Chi-Mei Optoelectronics (Taiwan) for the financial support.

\section{References}

1. D. K. Yang and S. T. Wu, Fundamentals of Liquid Crystal Devices (Wiley, 2006).

2. D. Armitage, I. Underwood, and S. T. Wu, Introduction to Microdisplays (Wiley, 2006).

3. J. M. Jonza, M. F. Weber, A. J. Ouderkirk, and C. A. Stover, "Polarizing beam-splitting optical component," U.S. Patent 5,962,114 (5 October, 1999).

4. G. P. Bryan-Brown and J. R. Sambles, "Polarization conversion through the excitation of surface plasmons on a metallic grating," J. Mod. Opt. 37, 1227-1232 (1990).

5. N. Passilly, K. Ventola, P. Karvinen, P. Laakkonen, J. Turunen, and J. Tervo, "Polarization conversion in conical diffraction by metallic and dielectric subwavelength gratings," Appl. Opt. 46, 4258-4265 (2007).

6. S. J. Elston, G. P. Bryan-Brown, T. W. Preist and J. R. Sambles, "Surface-resonance polarization conversion mediated by broken surface symmetry," Phys. Rev. B 44, 3483-3485 (1991).

7. Y. L. Kok and N. C. Gallagher, "Relative phases of electromagnetic waves diffracted by a perfectly conducting rectangulargrooved grating," J. Opt. Soc. Am. A 5, 65-73 (1988).

8. C. W. Haggans, L. Li, and T. Fujita, "Lamellar gratings as polarization components for specularly reflected beams," J. Mod. Opt. 40, 675-686 (1993).

9. S. R. Seshadri, "Polarization conversion by reflection in a thinfilm grating," J. Opt. Soc. Am. A 18, 1765-1776 (2001).

10. I. R. Hooper and J. R. Sambles, "Surface plasmon polaritons on narrow-ridged short-pitch metal gratings in the conical mount," J. Opt. Soc. Am. A 20, 836-843 (2003).

11. I. R. Hooper and J. R. Sambles, "Broadband polarizationconverting mirror for the visible region of the spectrum," Opt. Lett. 27, 2152-2154 (2002).

12. C. C. Tsai and S. T. Wu, "Study of broadband polarization conversion with metallic surface-relief gratings by rigorous coupled-wave analysis," J. Opt. Soc. Am. A (to be published)

13. A. V. Kats and I. S. Spevak, "Analytical theory of resonance diffraction and transformation of light polarization," Phys. Rev. B 65, 195406 (2002).

14. I. R. Hooper and J. R. Sambles, "Surface plasmon polaritons on a narrow-ridged short-pitch metal gratings," Phys. Rev. B 66, 205408 (2002). 
15. J. Chandezon, M. T. Dupuis, G. Cornet, and D. Maystre, "Multicoated gratings - a differential formalism applicable in the entire optical region,” J. Opt. Soc. Am. A 72, 839-846 (1982).

16. Y. Okuno, "The mode-matching method," in Analysis Methods for Electromagnetic Wave Problems, E. Yamashita, ed. (Artech House, 1990), pp. 107-138.

17. T. Suyama, Y. Okuno, and T. Matsuda, "Enhancement of TM-TE mode conversion caused by excitation of surface plasmons on a metal grating and its application for refractive index measurement," Prog. Electromagn. Res. PIER 72, 91-103 (2007).

18. M. G. Moharam, E. B. Grann, D. A. Pommet, and T. K. Gaylord, "Formulation for stable and efficient implementation of the rigorous coupled-wave analysis of binary gratings," J. Opt. Soc. Am. A 12, 1068-1076 (1995).
19. M. G. Moharam, D. A. Pommet, E. B. Grann, and T. K. Gaylord, "Stable implementation of the rigorous coupled-wave analysis for surface-relief gratings: enhanced transmittance matrix approach,” J. Opt. Soc. Am. A 12, 1077-1086 (1995).

20. P. C. Logofatu, S. A. Coulombe, B. K. Minhas, and J. R. McNeil, "Identity of the cross-reflection coefficients for symmetric surface-relief gratings,” J. Opt. Soc. Am. A 16, 1108-1114 (1999).

21. D.-E. Yi, Y.-B. Yan, H.-T. Liu, S. Lu, and G.-F Jin, "Broadband achromatic phase retarder by subwavelength grating," Opt. Commun. 227, 49-55 (2003).

22. E. D. Palik, Handbook of Optical Constants (Academic, 1997). 